\title{
DOS LIENZOS IDENTIFICADOS DE PIETER CRIJNSE VOLMARIJN EN EL ERMITAGE DE SAN PETERSBURGO Y GALERÍA DE LOS UFFIZI DE FLORENCIA
}

\author{
MATÍAs DíAz PADRÓN \\ Museo Nacional del Prado
}

\begin{abstract}
Pieter Crijnse Volmarijn (1612-1657) es un pintor enigmático y poco estudiado, limitado a dos artículos de los profesores J. S. Held y A. d'Hulst. El análisis estilístico nos lleva a la identificación de dos nuevas obras, una catalogada en recientes estudios de N. Gritsay y N. Babina como anónima, y la segunda incluida en la reciente monografía de Thomas Willeboirts Bosschaert. Este artículo aporta claves precisas para el estudio de la personalidad de Volmarijn y alguna consideración iconográfica.

Palabras clave: Pintura Flamenca; Siglo XVII; Jacob Jordaens; Thomas Willeboirts Bosschaert; Tres Reyes Magos; Pecadores arrepentidos.
\end{abstract}

\section{TWO PAINTINGS BY PIETER CRIJNSE VOLMARIJN IDENTIFIED IN THE HERMITAGE MUSEUM IN SAINT PETERSBURG AND THE GALLERY OF THE UFFIZI IN FLORENCE}

Pieter Crijnse Volmarijn (1612-1657) is an enigmatic, little-studied painter, limited to two articles by professors J. S. Held and A. d'Hulst. Stylistic analysis has led to the identification of two new works, one catalogued as anonymous in recent studies by N. Gritsay and N. Babina, and the other included in the recent monograph on Thomas Willeboirts Bosschaert. This article offers precise keys for the study of the personality of Volmarijn and certain iconographic considerations.

Key words: Flemish Painting; 17 $7^{\text {th }}$ century; Jacob Jordaens; Thomas Willeboirts Bosschaert; Three Kings; repentant sinners.

Estimo oportuno la propuesta a los pinceles de Pieter Crijnse Volmarijn del lienzo de los Tres Reyes Magos del Ermitage de San Petersburgo, catalogado entre los anónimos flamencos en la última revisión de los fondos del prestigioso museo ruso ${ }^{1}$. Los tres Reyes forman un grupo compacto con sus respectivos símbolos. El Rey de tez morena está en el centro de la composición con la mirada en éxtasis. Es el más joven en contraposición a Melchor y Gaspar con barba blanca uno y cabellos negros el otro en armónico diseño a los lados. Los tres dominan el primer plano (fig. 1).

El silencio a su autoría en el último catálogo del Ermitage no está reñido con las referencias documentales a su origen más inmediato y referencia crítica en catálogos anteriores. Su autoría ha estado siempre en tela de juicio. La primera noticia registra la última catalogación en la colección de Lev Zubalov en Moscú en 1914, de donde pasó al Rumyantsev museum en 1931, habiendo sido adquirido en el mercado de antigüedades; pero pienso que con anterioridad fue de la colección Bernatzky en 1905, y atribuido a Jacob Jordaens en venta en Colonia $^{2}$. Nos adelantamos a reconocer un mismo lienzo, no réplica como duda el catálogo del Ermitage. Existen ligeras diferencias en las dimensiones transcritas con el catálogo de Colonia, pero esto puede deberse a la falta de precisión en la medición. Las diferencias son escasas. A esto se une la superficie oscura en la fotografía de 1905 por acumulación de excrecencias y barniz oxidado. Un atento análisis lleva a pensar que se trata de la misma obra.

La atribución a Jordaens y círculo de las antiguas referencias del Ermitage se pone en duda en el catálogo de $1958^{3}$, que la atribuye a Pierre-Joseph Verhagen por comunicación verbal de

\footnotetext{
${ }^{1}$ Gritsay, Natalya \& Babina, Natalya, State Hermitage Museum Catalogue, Seventeenth and Eighteenth-Century Flemish Painting, San Petersburgo, 2008, n. ${ }^{\circ}$ 540, p. 437.

${ }^{2}$ Herbelé, Lempertz Cologne von Bernatzky 10-X-1905, lot.18.

3 Gosudarstvennyy Ermitaz. Otdel Zapadnoevropeyskogo iskusstva. Katalog zhivopisi, 1958, p. 45.
} 


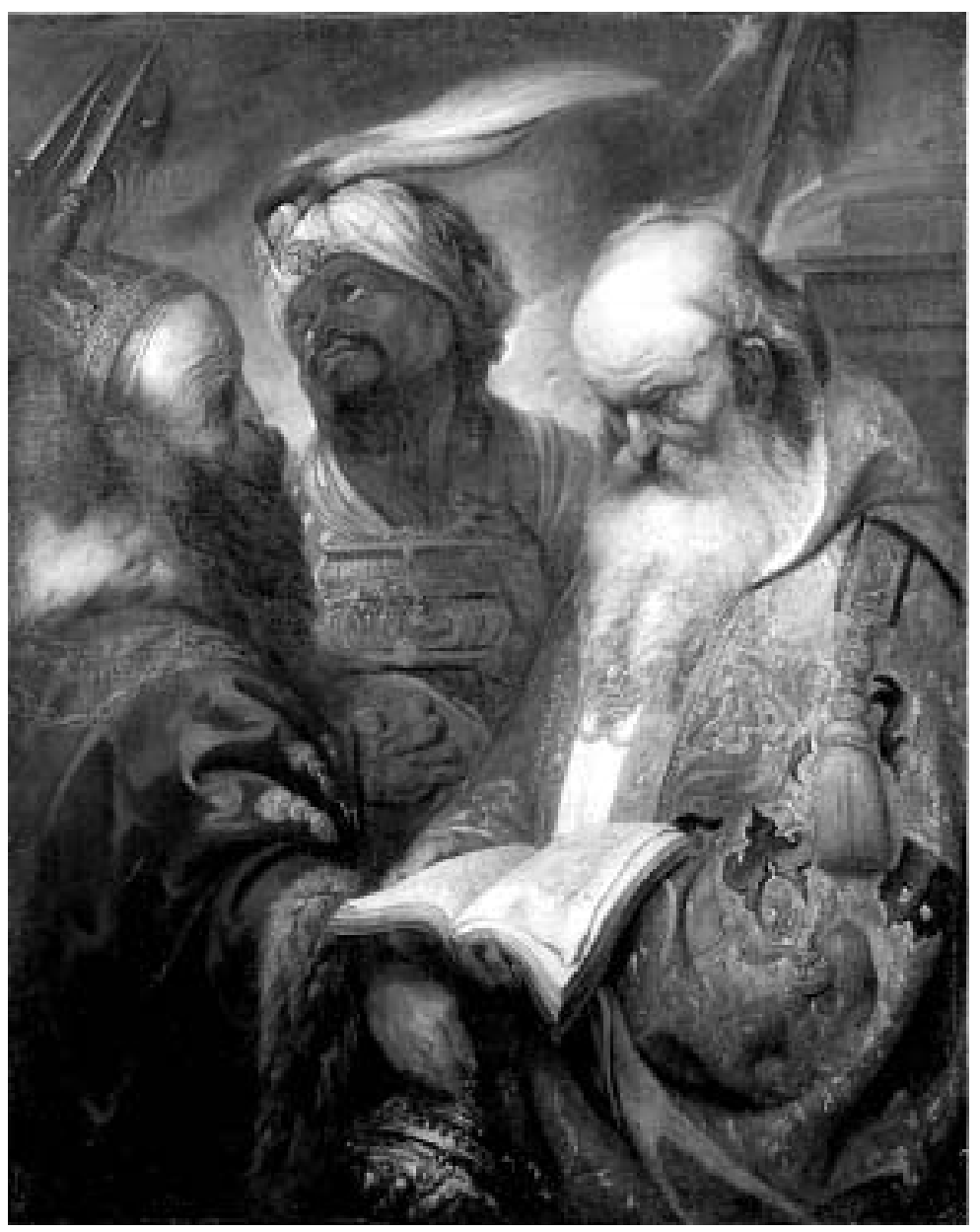

Fig. 1. Pieter Crijnse Volmarijn, Los tres Reyes Magos, Museo del Ermitage, San Petersburgo.

M. I. Shcherbachova, recogida por Asaevich en $1970^{4}$. Basa esta atribución por cotejo con la Adoración de los Magos del museo Vander Kelen-Mertens de Lovaina. Propuesta que rechaza razonablemente el catálogo del museo de $1981^{5}$, inscribiéndolo entre los anónimos de escuela flamenca del siglo XVII, opinión rubricada por N. P. Babina y N. I. Gritsay en $2005^{6}$, y más reciente edición ${ }^{7}$, marcando las diferencias con la estética del pintor entrado el siglo XVIII como P. J. Verhagen, a pesar de la huella con el siglo XVII.

Hasta aquí la cuestión crítica de este lienzo que restituimos a Pieter Crijnse Volmarijn. Es comprensible la dificultad en identificar su autoría, pues su obra es escasa, y fruto en los últi-

\footnotetext{
${ }^{4}$ Asaevich, K. F., "P'yer Zhozef Verkhagen i Andreas Lens i ikh proizvedeniya v Ermitazhe" en Zapadnoevropeyskoe iskusstvo, Leningrado, 1970, p. 203.

${ }^{5}$ Gosudarstvennyy Ermitaz Zapadnoevropeyskaya zhivopis, p. 56.

${ }^{6}$ BABINA \& GRITSAY, Gosudarstvennyy Ermitazh, Flamandskaya zhivopis' XVII-XVIII vekov. Katalog kolletsii, 2005, n. ${ }^{\circ} 260$, p. 258.

7 Véase nota 1.
} 
mos años de exclusivos artículos de los profesores A. D'Hulst ${ }^{8}$ y J. S. Held ${ }^{9}$, en publicaciones de difícil acceso. Hasta el punto de pasar por anónimo discípulo de Jordaens el Galeno visitando a una mujer enferma, en la antigua colección Goudstikker, expuesta en la galería Christie's de Amsterdam (14 noviembre 2007, lot. 39). Esta pequeña tabla estaba estudiada y correctamente catalogada por el profesor d'Hulst en $1970^{10}$. Hasta entonces el nombre de Pieter Crijnse Volmarijn figuraba sólo con escueta referencia y fechas (1612-1657) en el ThiemeBecker ${ }^{11}$

Aunque nació en Róterdam (al norte de los Países Bajos), su estilo está impregnado de la influencia de Rubens, Jordaens y Van Dyck en la escuela de Amberes de la primera mitad del siglo XVII. No es difícil reconocer en la imagen de Melchor con barba blanca a San Amando leyendo un grueso libro en la puerta izquierda del tríptico del Alzamiento de la Cruz de Rubens en la catedral de Amberes. Presta atención a la lectura del libro y su plástica monumentalidad. El lujo lo acentúa igual en los brocados de la túnica. Más próximo está Santo Tomás del Apostolado del Museo del Prado (n. ${ }^{\circ}$ 1654) (fig. 2) y estudio de la colección L. Koetser Gallery de Londres. También el rostro del Rey negro es familiar en las cabezas de Rubens del museo de Bruselas (n. ${ }^{\circ}$ 3176) y Aquiles descubierto por Ulises y Nicomedes del Museo del Prado. Más personal es el rostro del Rey de barba negra del lado izquierdo de acusado naturalismo. Advertimos aquí la característica más singular de este pintor, inclinado a la individualidad expresiva de sus personajes. Los tres Reyes dominan el primer plano con notorio horror al vacío.

La deformación de los personajes está al límite de un singular expresionismo acorde al espíritu nórdico. El desbordar las formas explica la frecuente confusión con J. Jordaens. En común tienen la monumentalidad y la deformación; pero P. C. Volmarijn perfila con fino diseño las siluetas, la anatomía del rostro y la decoración de los brocados de sus pesadas telas. La densidad formal no rompe la delicadeza en los pormenores y detalles suntuarios: regalos de oro, incienso y mirra que los tres Reyes portan en el lienzo que tratamos. Muy personal es la sombra envolvente en misterio sin la dureza de Rubens del periodo clásico. Asume P. C. Volmarijn un raro sincretismo con la tradición holandesa del circuito rembrandtiano.

Los Tres Reyes solos, no es frecuente en la iconografía. Las lanzas que asoman al lado izquierdo parecen avisar el encuentro de los Magos con el rey Herodes y su guardia. Quizá la mirada a lo alto de Baltasar es premonición a la vista de la estrella que servirá de guía, "porque hemos visto la estrella al Oriente, y vamos a adorarle" (Mateo, II, 1-2). El Rey de negra barba lleva un incensario ricamente repujado igual que la caja de mirra de Baltasar; pero el libro no es habitual en los tres Reyes. Pensamos que se asocia con los libros de astrología y ciencia

${ }^{8}$ D’Hulst, A., "Pieter Crijnse Volmarijn, een Rotterdamse Navolger van de Antwerpse Schilderschool uit de 17 de Eeuw", Mededelingen van de Koninklijke Vlaamse Academie voor Wetenschappen, Letteren en Schone Kunsten van België, 32 (Bruselas, 1970), p. 1; Idem, "Een paar toevoegingen aan het oeuvre van Pieter Crijnse Volmarijn", en Essays in Northern European art (E. Haverkamp-Begemann), 1983, p. 113.

9 Held, J. S., "Further Data on Pieter Crijnse Volmarijn", en Essays in Northern European art (E. HaverkampBegemann), 1983, p. 102.

10 D'Hulst, op. cit., 1970, p. 17.

11 ThiEme-Becher, Allgemeines Lexikon der Bildenden Künstler von der Antike bis zur Gegenwart (1907-1950), 1940, tomo 34, p. 530; recordamos ocasional referencia en Gudlaugsson, S. J., "Crijn Hendricksz Volmarijn, een Rotterdamse Caravaggist", oud Holland, 67 (1952), p. 241, y SCHADEE, N. (red.), Rotterdamse Meesters uit de Gouden Eeuw, Rotterdam (Historisch Museum), 1994, p. 307. Y recientemente en la Galería Christie's (27-ene-2009, lot 31), correctamente identificada a Pieter Crijnse Volmarijn, aquí con clara influencia de Rubens. Ha sido frecuente la confusión de Pieter Crijnse Volmarijn con Th. Willeboirts Boschaert (HeInRICH, Axel, Thomas Willeboirts Bosschaert

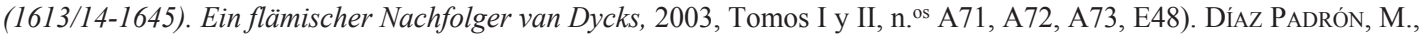
"Nuevas obras de Pieter Crijnse Volmarijn identificadas" (artículo en preparación). 


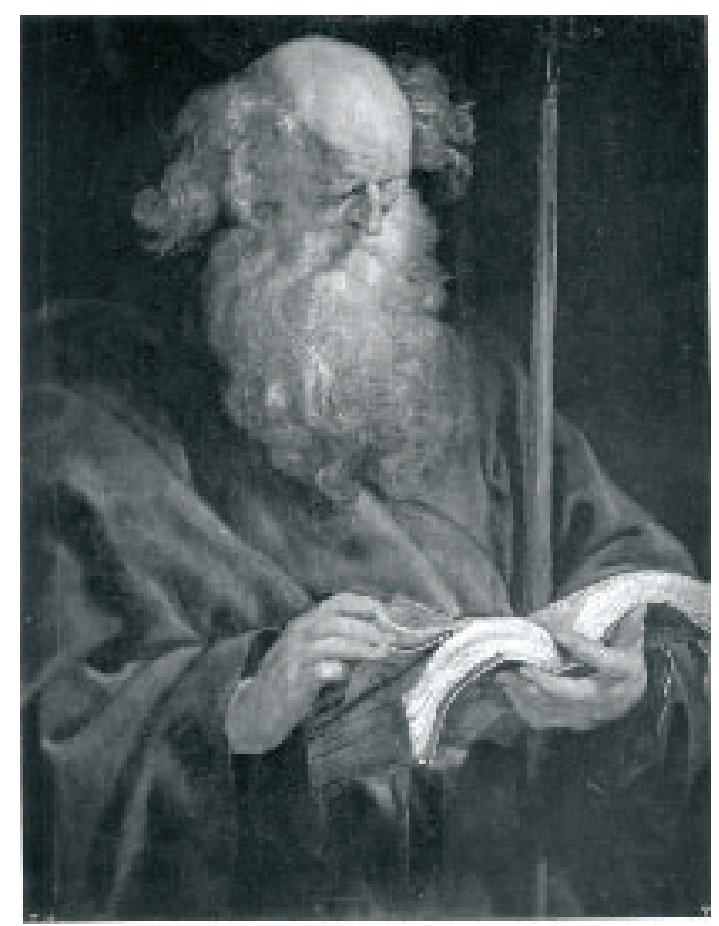

Fig. 2. P. P. Rubens, Santo Tomás, Museo Nacional del Prado, Madrid.

cultivados por los sacerdotes que vienen de la mítica Babilonia, transmisores de presagios de los astros y su influencia en el destino de los hombres.

El segundo lienzo, La Virgen y los Santos Pecadores de la Galería de los Uffizi de Florencia (fig. 3), lo publicó Marco Charini por original de Thomas Willeboirts Bosschaert ${ }^{12}$, y Axel Heinrich en la reciente monografía de este excelente seguidor de Van Dyck ${ }^{13}$. El primero de los trabajos registra el origen en la galería de Viena, de donde pasa a Florencia con atribución a Teodoro Rombouts y como Alegoría Sacra. Didier Bodart lo cita en la exposición de Homenaje a Rubens de 1977 entre las pinturas destruidas en 1944 sin documentación fotográfica. En el inventario de 1890 se atribuyó a la escuela de Van Dyck y desaparece del depósito de Pistoia donde estaba desde $1929^{14}$.

La catalogación como Virgen con Niño en la Gloria y Alegoría de la Redención, pueden sustituirse por La Virgen con el Niño y los Pecadores arrepentidos. María Magdalena y el rey David flanquean el escenario, San Pedro aparece con su calvicie bañada por la luz que emana del rompimiento de gloria deslizando sus rayos en el Niño, la Virgen y la Magdalena. Los restantes quedan en penumbra. El rey David de perfil a contraluz es identificable por la corona y el arpa. En el fondo reconocemos con alguna dificultad al pecador arrepentido, Simón el fariseo y el hijo pródigo entre sombras con intención de resaltar a la Virgen y la Magdalena. Un pesado cortinaje desplegado a los lados da paso al rompimiento de gloria con angelitos en dinámica diagonal.

Es una espectacular visión teatral con columna en el fondo, borlas y brillos de la esfera, la corona, y el vaso de ungüentos que lleva la Magdalena. La composición está inscrita bajo un triángulo ascendente con efectismo manierista. Recurso estético frecuente en este imprevisible pintor revivido.

El tema de los pecadores arrepentidos está más divulgado en la pintura flamenca del siglo de Rubens con Van Dyck y Gerard Seghers que en otros países católicos ${ }^{15}$. Es una visión a favor del dogma más detestado por los reformistas protestantes: "Para los católicos salvar las almas del

12 Chiarini, M., “Un'Allegoria della redenziones di Thomas Willeboirts Bosschaert agli Uffizi”, Bollettino d'Arte del Ministero per $i$ Beni Culturali e Ambientali, n. ${ }^{\circ}$ 5, enero-marzo 1980, p. 85.

13 HernRICH, A., Thomas Willeboirts Bosschaert (1613/14-1645) Ein flämischer Nachfolger van Dycks, 2003, Tomo I, p. 262, n. ${ }^{\circ}$ A73.

14 Bodart, D., Rubens e la Pittura fliaminga del Seicento, Florencia, Palacio Pitti, 22-7-/9-10-1977, p. 336. Proviene de la galería de Viena desde el 15 de diciembre de 1793, estuvo atribuida en 1890 a la escuela de Van Dyck; veáse además: Cat. de Florencia de 1910, p. 143, n. ${ }^{\circ}$ 150, Cat. Florencia 1979, p. 592, n. ${ }^{\circ}$ P1898.

15 KNIPPING, J. B., Iconography of the Counter Reformation in the Netherlands, 1974, p. 317. 


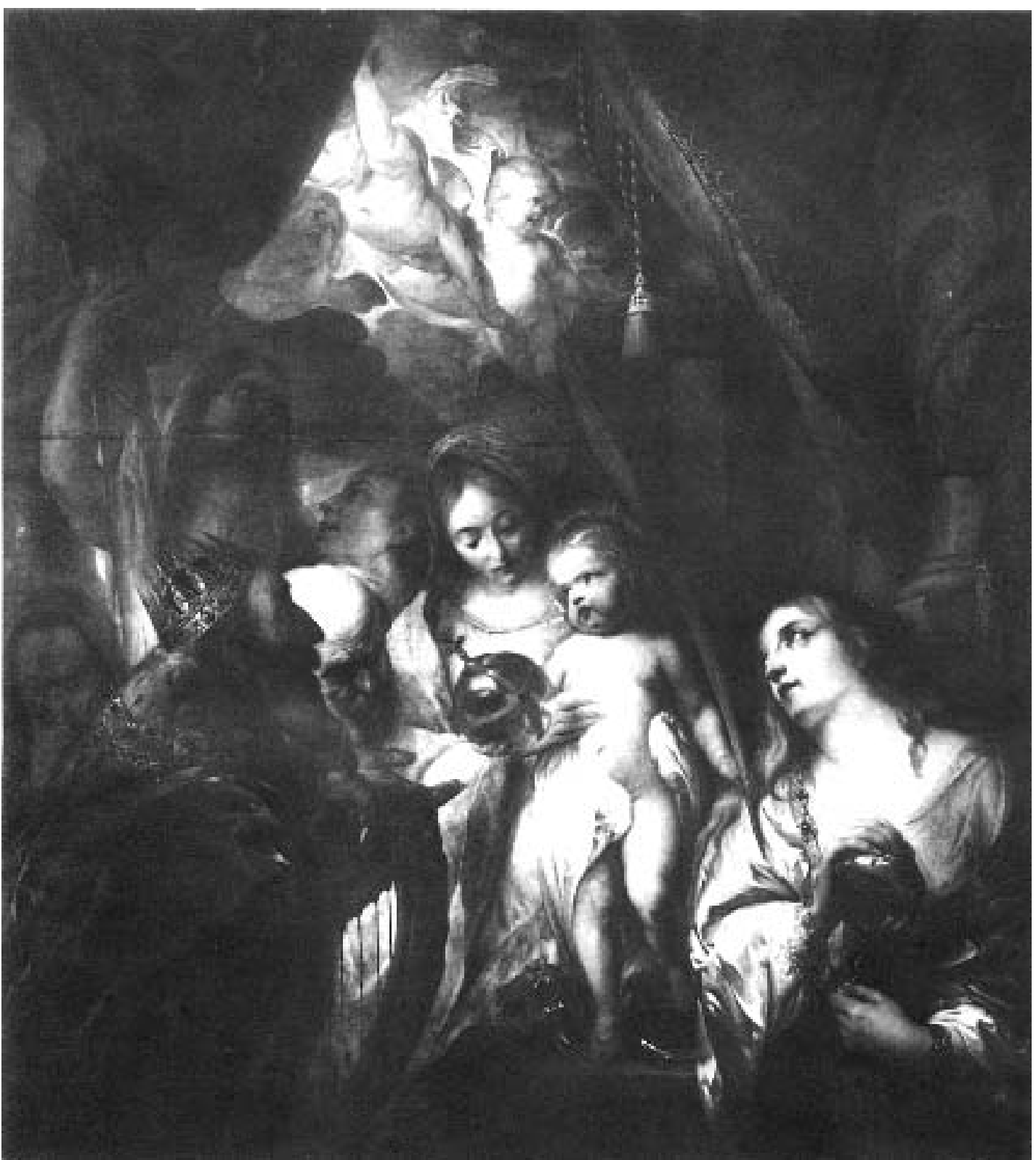

Fig. 3. Pieter Crijnse Volmarijn, La Virgen y el Niño con los santos Pecadores arrepentidos, Galería de los Uffizi de Florencia. 


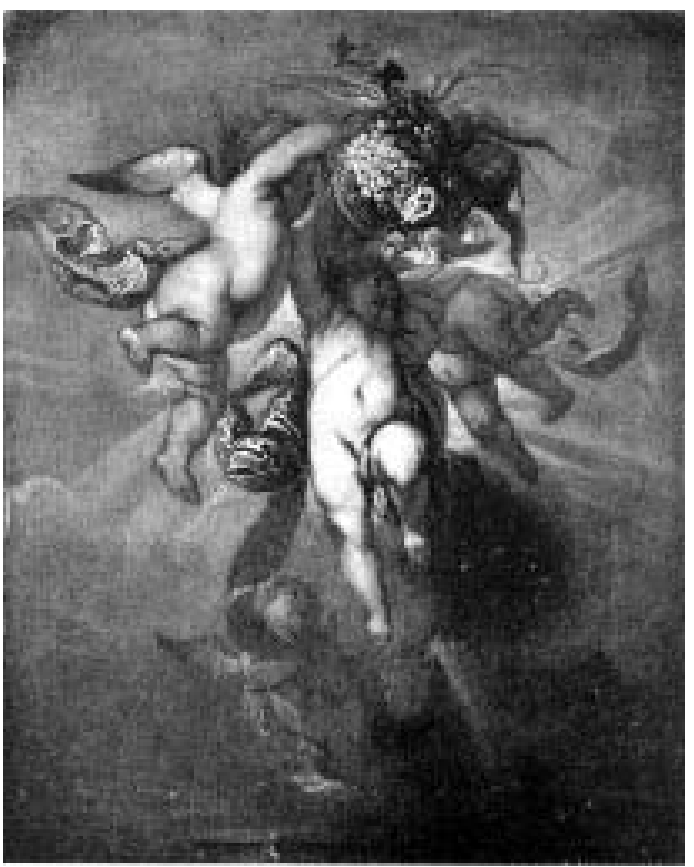

Fig. 4. P. C. Volmarijn, Putti con el cuerno de la abundancia, Galería Fiévez Bruselas.

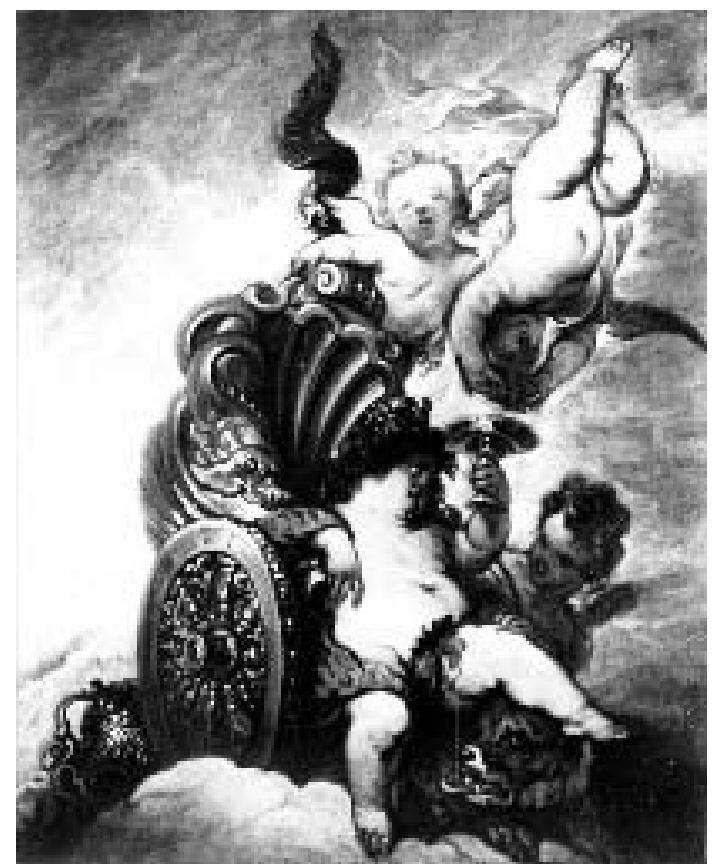

Fig. 5. P. C. Volmarijn, Joven Baco, colección privada de Estocolmo.

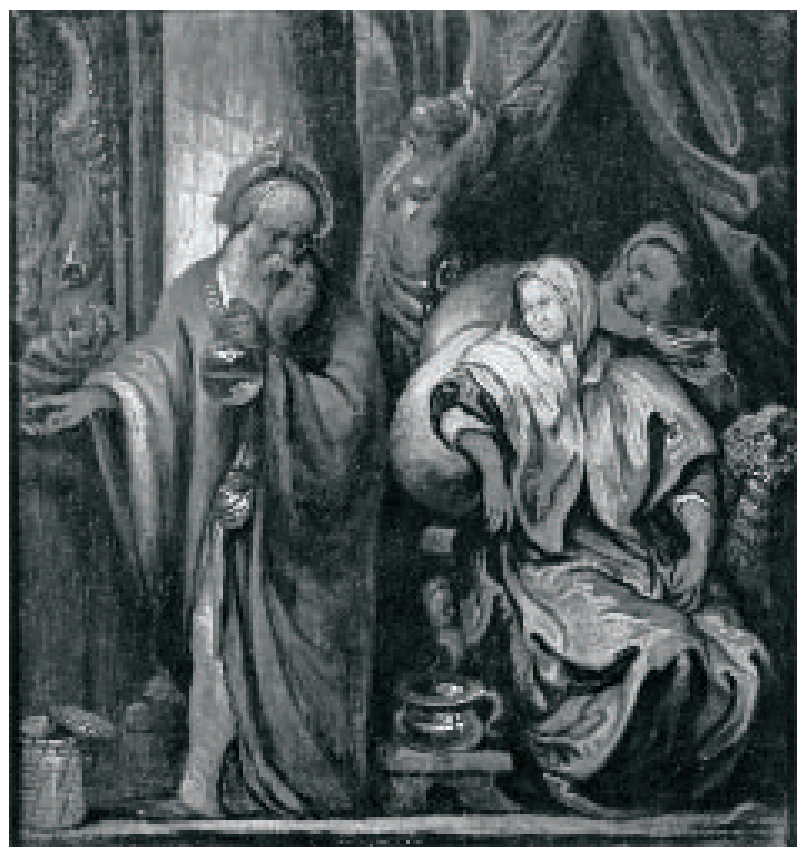

Fig. 6. P. C. Volmarijn, Galeno atendiendo a una joven enferma, col. priv. Barcelona. 
purgatorio es cosa santa y rezar por los muertos para que sean salvados de los pecados"16. Es evidente el impacto de los jesuitas defensores de este sentimiento en los Países Bajos ${ }^{17}$. En el lienzo destaca la imagen de la Magdalena con los ungüentos y David suplicando perdón ${ }^{18}$. Veo la composición liberada de la influencia de los maestros citados. Es original la luz y las fugas diagonales. David a contraluz contribuye a la emoción del acto, sin romper la unidad del conjunto en penumbra. El tercer personaje toma la luz de lo alto en su calva. Acentúa la dinámica el ramillete de angelitos en diagonal ascendente. Muchos son familiares en pinturas y dibujos del pintor ${ }^{19}$ (figs. 4 y 5). Es posible la sugestión de Van Dyck en La Virgen y el Niño del Fitzwilliam Museum de Cambridge, y de Rubens. Aquí la Virgen baja la cabeza con tristeza, mientras el Niño dirige su mirada al rey. La vivacidad de sus pupilas y la frente acusada es típica en los niños de Pieter Crijnse Volmarijn. Interesante es para nosotros el diseño del rey David. El perfil es el mismo de Gaspar, en los Tres Reyes Magos del Ermitage que hemos estudiado. Incluso, la corona de formato cónico es igual en uno y otro lienzo. También la cadena y la piel de armiño en la espalda. El brillo de los ornamentos en la penumbra nos avisa del sutil contacto con el ambiente rembrandtiano. El personaje con la calva que identificamos con San Pedro repite el modelo del Galeno atendiendo a una mujer enferma de la antigua colección Goudstikker y hoy en colección privada de Barcelona (fig. 6) ${ }^{20}$.

A estas alturas vemos comprensible la reflexión de Julius Held al publicar nuevas obras del pintor que tratamos, reconociendo la dificultad de su estudio. Había reunido años atrás algunas obras, admitiendo la compleja personalidad de este "pintor travieso que ha dado muchos quebraderos de cabeza a los estudiosos de la pintura flamenca" 21.

\footnotetext{
16 MÂLE, E., L'Art religieux après le Concile de Trente, 1951, p. 58.

17 De Cartagena, Juan, Homilias, IV, De Mirandis Deiparae Virginia, p. 228 [cit. MÂle, op. cit., 1951, p. 61].

18 MÂLE, E., L'Art religieux après le concile de Trente, París, 1951, id., p. 69; RÉAU, L., Iconographie de l'art chrétien, París, 1957, t. II, Libro 1. ${ }^{\circ}$, Antiguo Testamento, p. 276.

19 D’Hulst, op. cit., 1970, p. 18, n. ${ }^{\circ} 13$ en galería Fiévez de Bruselas (1930) y p. 19, n. ${ }^{\circ} 14$ en colección privada de Estocolmo (1938); D’Hulst, op. cit., 1983, p. 122, n. ${ }^{\circ} 4$.

${ }^{20}$ En catálogo reciente de la galería Christie's de Amsterdam se cataloga como seguidor de Jacob Jordaens (14Nov-2007, n. $\left.{ }^{\circ} 39\right)$ sin conocer su catalogación muy anterior y correcta de D’Hulst, op. cit., 1970, p. 17.

${ }^{21}$ HeLD, J. S., 1983, op. cit., p. 102.
} 